\title{
PROGRAMMING FOR DIGITAL COMPUTATION OF SUSPENSION BRIDGES UNDER VERTICAL, HORIZONTAL AND TORSIONAL LOADINGS
}

\author{
By Shizuo Shimada*, C.E. Member
}

\section{Introduction}

A treatment is introduced over "how-to" utilize the electronic digital computers for the calculation of suspension bridges under horizontal and torsional forces as well as vertical loadings.

Because of non-linearity of suspension bridges on the spatial deformation, statical values such as bending moments of stiffening girder are not easily determined that we may operate manual calculs.

Equilibrium equations for the structure are three differential equations on the deflection $v$, the horizontal displacement $u$, and the torsional deformations $\varphi$ of the stiffning girder, and they are not independent each other, in other words, each equation shows non-linear differential one including every other variable such as $u, v, \varphi$ and various static values. Some elastic equations are also to be considered in order to accomodate elastic conditions for cables.

Problems are to solve these equations and to obtain the numerical values for given suspension bridge. When we use the electronic digital computers, it is the most important task that how to compose the programme of calculation.

Since the most of the engineers who are concerned with the structural mechanique have few chances really to operate the digital computers by themselves, and operaters or programmers of the computers also know few knowledge about the real meaning of the equations, so previous treatment of equations is necessary before they are undertaken by the programmers.

The previous treatment is, in short, to compose the calculating flow chart and to give the practical calculating formulas instead of analy-

\footnotetext{
* Dr. of Eng. Lecturer, Department of Civil Enginee-
}

ring, University of Tokyo. tical display. When we are to utilize the digital computers, usual theoretical and analytical results and methods of calculs are not always suitable, we must therefore serch somewhat different sort of calculating methods.

The author introduces in the following articles how to compose the flow chart for the calculation of the vertical, horizontal and torsional behaviours of suspension bridge. In the second article, theoretical and analytical presentation is dealed. The third article shows the modification of such theoretical equations to practical formulas to fit in the practical solutions. The last article gives the conclusive results of the flow chart. This article be sent to any operators or programmers, we would obtain the values of given suspension bridge without any explanation.

\section{Fundamental equations}

Let a suspension bridge be considered as are shown in Figs. 1 and 2 with definitions of

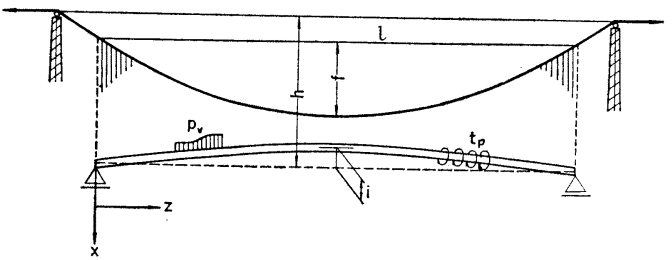

Fig. 1

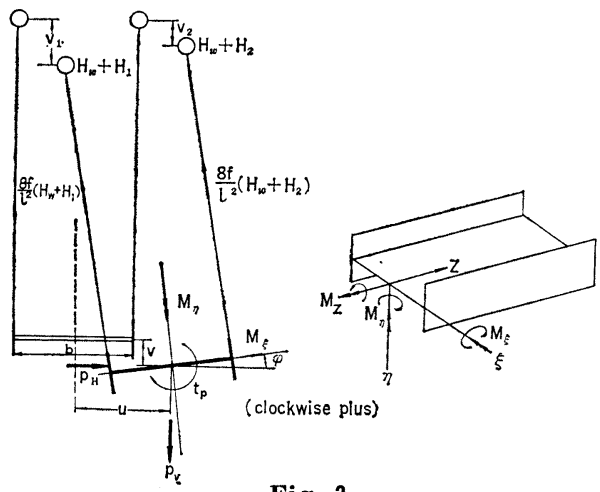

Fig. 2 
spatial co-ordinates, and of directions of deformations and forces. Two parallel cables are to carry total weight of structures by the infinitely close spaced curtain-like suspenders causing no stresses in the stiffning girder under the standard state, that is, there are no loadings and under usual temperature.

The shapes of the cables and the stiffning girder are to be defined as eqs. (1) and (2), wherein the cables take a symmetric curve of parabola and the stiffning girder is to have the initially logitudinal parabolic camber.

$$
\begin{aligned}
& Y_{c}=\frac{4 f}{l^{2}} z(l-z) \cdots \\
& Y_{J}=h-\frac{4 i}{l^{2}} z(l-z)
\end{aligned}
$$

The deflection $v$ and torsional angle $\varphi$ of the stiffning girder cause the deflection $v_{1}$, and $v_{2}$ of the left and right cables, respectively.

$$
\left.\begin{array}{l}
v_{1}=v-b \varphi / 2 \\
v_{2}=v+b \varphi / 2
\end{array}\right\}
$$

Each cable then varies its tension. Usually we consider the horizontal reaction of tensile forces that varies from $H_{w}$ to $\left(H_{w}+H_{1}\right)$ and $\left(H_{w}+H_{2}\right)$ for each cable. By the close spaced suspenders, each cable gives the distributing oad $p_{1}$ and $p_{2}$ to the stiffning girder.

$$
\left.\begin{array}{l}
p_{1}=\left(H_{w}+H_{1}\right) \frac{d^{2}}{d z^{2}}\left(Y_{c}+v_{1}\right) \\
p_{2}=\left(H_{w}+H_{2}\right) \frac{d^{2}}{d z^{2}}\left(Y_{c}+v_{2}\right)
\end{array}\right)
$$

As $p_{1}$ and $p_{2}$ work along the left and right side of the stiffning girder, they are composed so as to cause the vertical and horizontal forces along the sectional gravity center and, the twisting moment around the shear center or twisting center of the stiffning girder.

External forces or loadings against the stiffning girder are, the vertical loadings $p_{v}$ like traffic weight, also the dead weight $p_{d}$ of stiffning girder itself, the horizontal forces $p_{H}$ like wind thrust, and the torque $t_{p}$ which would occure when the traffic loads do not lie just on the center axis of bridge width or when wind thrust works so as to make a torque around the shear center.

The stiffning girder is then affected under the three component of forces as shown eqs. (5)

$$
\left.\begin{array}{ll}
\text { vertical } & p_{v}+p_{d}-\left(p_{1}+p_{2}\right) \\
\text { horizontal } & p_{H}-\theta\left(p_{1}+p_{2}\right) \\
\text { torque } & t_{p}+\frac{b}{2}\left(p_{1}-p_{2}\right)
\end{array}\right\}
$$

where $\theta$ is the slope angle of the suspenders, and it may be allowed to assume nearly equal to the ratio $u / h$. The dead weight $p_{d}$ balances to the cable reaction as eq. (6)

$$
p_{d}=-2 H_{w} \frac{d^{2} Y_{c}}{d z^{2}}=2 \cdot \frac{8 f}{l^{2}} \cdot H_{w}
$$

Succeedingly, we consider the deformation and static values of the stiffning girder itself. Sectional bending moments around the two principal axis $\xi, \eta$ and torsional moment around the longitudinal axis are;

$$
\begin{aligned}
& M_{\xi}=-E J_{\xi}\left[\frac{d^{2} v}{d z^{2}}-\frac{d^{2} u}{d z^{2}} \varphi\right] \\
& M_{\eta}=-E J_{\eta}\left[\frac{d^{2} u}{d z^{2}}+\frac{d^{2} v}{d z^{2}} \varphi\right] \\
& M_{z}=-E C \frac{d^{3} \varphi}{d z^{3}}+G K \frac{d \varphi}{d z}
\end{aligned}
$$

As for the reduced components inside the vertical and horizontal plain, we obtain from $M_{\xi}$ and $M_{\eta}$,

$$
\left.\begin{array}{l}
M_{x}=-E J_{\xi} \frac{d^{2} v}{d z^{2}}+\left(E J_{\xi}-E J_{\eta}\right) \varphi \frac{d^{2} u}{d z^{2}} \\
M_{y}=-E J_{\eta} \frac{d^{2} u}{d z^{2}}+\left(E J_{\xi}-E J_{\eta}\right) \varphi \frac{d^{2} u}{d z^{2}}
\end{array}\right\}
$$

As the axis of the stiffning girder runs with the spatial curvature, bending moments $M_{x}$ and $M_{y}$ cause the additional torque around the axis;

$$
t(z)=\frac{d^{2} u}{d z^{2}} M_{x}-\left(\frac{d^{2} Y_{J}}{d z^{2}}+\frac{d^{2} v}{d z^{2}}\right) M_{y} \cdots(9)
$$

Differentiate the eqs. (8) twice by $z$, and the $M_{z}$ of eq. (7) once, and we obtain three fundamental differential equations upon $u, v$ and $\varphi$. Considering eqs. (5) and (9), and assuming the shear center of the section is not so deviated from the gravity center, we get;

$$
\begin{aligned}
& E J_{\xi} \frac{d^{4} v}{d z^{4}}-\left(E J_{\xi}-E J_{\eta}\right) \frac{d^{2}}{d z^{2}}\left[\varphi \frac{d^{2} u}{d z^{2}}\right]=p_{V_{1}} \\
&+\left(2 H_{W}+H_{1}+H_{2}\right) \frac{d^{2} v}{d z^{2}}+\frac{b}{2}\left(H_{2}-H_{1}\right) \\
& \times \frac{d^{2} \varphi}{d z^{2}}-\left(H_{1}+H_{2}\right) \frac{8 f}{l^{2}} \cdots \cdots \cdots \cdots \cdots \\
& E J_{\eta} \frac{d^{4} u}{d z^{4}}-\left(E J_{\xi}-E J_{\eta}\right) \frac{d^{2}}{d z^{2}}\left[\varphi \frac{d^{2} v}{d z^{2}}\right]=p_{H}
\end{aligned}
$$

